\title{
DÜBLIN
}

Technological University Dublin ARROW@TU Dublin

2004-01-01

\section{Objective Measurements of Image Quality}

\author{
Jacinta Browne \\ Technological University Dublin, jacinta.browne@tudublin.ie \\ Amanda Watson \\ Western Infirmary Glasgow \\ Nicholas Gibson \\ Nottingham City Hospital
}

See next page for additional authors

Follow this and additional works at: https://arrow.tudublin.ie/scschphyart

Part of the Physics Commons

\section{Recommended Citation}

Browne, J., Watson, A., Gibson, N., Dudley, N., Elliott, A.: Objective measurements of image quality. Ultrasound in Medicine and Biology, Vol.30 (2), pp. 229-237. 2004. doi:10.1016/

j.ultrasmedbio.2003.10.002

This Article is brought to you for free and open access by the School of Physics \& Clinical \& Optometric Science at ARROW@TU Dublin. It has been accepted for inclusion in Articles by an authorized administrator of ARROW@TU Dublin. For more information, please contact arrow.admin@tudublin.ie, aisling.coyne@tudublin.ie, gerard.connolly@tudublin.ie.

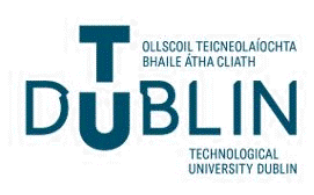




\section{Authors}

Jacinta Browne, Amanda Watson, Nicholas Gibson, Nicholas Dudley, and Alex Elliott

This article is available at ARROW@TU Dublin: https://arrow.tudublin.ie/scschphyart/6 


\section{OBJECTIVE MEASUREMENTS OF IMAGE QUALITY}

\section{JACINTA E BROWNE ${ }^{1}$, AMANDA J WATSON ${ }^{1}$, NICHOLAS M GIBSON ${ }^{2}$, NICHOLAS J DUDLEY ${ }^{2}$ AND ALEX T ELLIOTT ${ }^{3}$}

Ultrasound Equipment Evaluation Project, Western Infirmary, Glasgow, UK ${ }^{1}$, Dept. of Medical Physics, Nottingham City Hospital, $\mathrm{UK}^{2}$, and Dept. of Clinical Physics, Western Infirmary, Glasgow, $\mathrm{UK}^{3}$.

Dr Jacinta Browne, Ultrasound Equipment Evaluation Project, Dept. of Clinical Physics, Ground Floor, 38 Church Street, Glasgow, G11 6NT, UK.

Tel: $0141-2116292$

Fax: $0141-2111772$

Email: jacinta.browne@northglasgow.scot.nhs.uk

Key Words: Computerised image analysis program, Tissue harmonic imaging, compound imaging, harmonic compound imaging, quality control. 


\section{OBJECTIVE MEASUREMENTS OF IMAGE QUALITY}

\section{Abstract:}

Tissue harmonic imaging (THI) and compound imaging have been reported clinically to improve contrast resolution, tissue differentiation and overall image quality. However, there have been limited studies to date to quantify objectively the improvements in image quality achieved with these new imaging techniques. The aim of this study was to quantify differences in image quality which exist between conventional B-mode imaging, harmonic imaging, compound imaging and harmonic compound imaging. An ATL HDI 5000 scanner with three probes (C5-2, L7-4 and L12-5) was tested with two different types of test object, the Gammex-RMI model 404 GS LE and the Gammex-RMI 403 GS LE. The measurement limitations associated with subjective analysis methods were not present in this study, since an automated image analysis program was used to determine the image quality parameters therefore, subtle differences between the four imaging modes could be detected. Significant improvements in lateral resolution and slice thickness as a function of depth were found with THI. Contrast resolution and anechoic target detection improved with compound imaging, while harmonic compound imaging improved lateral resolution, slice thickness as a function of depth and contrast resolution. (E-mail: Jacinta.browne@northglasgow.scot.nhs.uk) 


\section{Introduction}

In recent years, diagnostic ultrasound image quality has greatly improved, due to advances in technology and introduction of new techniques, such as compound imaging and tissue harmonic imaging (Claudon et al 2002). Developments in transducer design have resulted in transducers with greater bandwidth and sensitivity (Averkiou et al 1997). Broadband transducers allow large bandwidth pulses to be generated, which is vital for pulse inversion tissue harmonic imaging and also leads to improvements in axial resolution. Tissue harmonic imaging (THI) is a real-time ultrasound imaging technique which uses echoes at twice the transmitted frequency to form the image, unlike conventional B-mode imaging which uses echoes at the transmitted frequency to form the image (Desser and Jeffrey 2001). The higher frequency harmonic signal is not present in the transmitted signal but is generated as it propagates through tissue due to the phenomenon of nonlinear sound propagation. Imaging with the harmonic signal means that the harmonic beam only passes once at echo reception through the body wall and any fat that is present (Desser and Jeffrey 2001). THI has been reported to improve image quality, particularly in clinical applications such as cardiology and abdominal imaging (Desser and Jeffrey 2001; Li and Zagzebski 2000; Tranquart et al 1999; Averkiou et al 1997; Christopher 1997; Ward et al 1997).

Real-time compound imaging has been made possible, only recently, due to the substantial computational power of modern all-digital ultrasound systems, despite the principles and benefits of it being known since the 1950s (Howry 1955). There are two types of compound imaging, spatial compound imaging and frequency compound 
imaging. Spatial compound imaging involves acquiring a number of coplanar images of the same object from different angles, and then combining them into a single compound image (Entrekin et al 2000). Frequency compounding involves acquiring images of the same object at different frequencies and then combining them into a single compound image (Gatenby et al 1989). Spatial compound imaging has been reported to improve contrast resolution, tissue differentiation and overall image quality in breast imaging, vascular imaging and musculoskeletal imaging (Entrekin et al 2001; Entrekin et al 2000; Jespersen et al 2000). Despite the above clinical studies being conducted to evaluate the improvements in image quality, there have been limited technical evaluations to provide quantitative information about the improvements in image quality with THI, compound imaging and the newer technique of harmonic compound imaging technique, combining both methods and introduced by ATLPhilips. The aim of this study was to quantify differences in image quality which exist between conventional B-mode imaging, THI, compound imaging and harmonic compound imaging.

\section{METHODS}

\section{Image Quality Tests}

The image quality parameters that were measured in the study were: (i) axial resolution; (ii) lateral resolution; (iii) slice thickness; (iv) contrast resolution; (v) anechoic target detection; and (vi) low contrast penetration depth. These image quality test parameters were chosen for testing the ultrasound scanner in order to obtain a full evaluation of its imaging capabilities, as recommended by a number of professional bodies (Price 1995; Goodsitt et al 1998). 


\section{Equipment}

The ultrasound scanner used in this study was the HDI 5000 (Philips/ATL, Bothell, USA), a high-end system, dedicated for general ultrasound examinations with a 2-5 MHz (C5-2) curvilinear transducer, a 4-7 MHz (L7-4) linear transducer and a 5-12 MHz (L12-5) linear transducer. In addition to conventional B-mode imaging, both the C5-2 and the L7-4 had tissue harmonic, compound and harmonic compound imaging available, while the L12-5 had only compound imaging. The tissue harmonic imaging (THI) mode on the HDI 5000 works by pulse inversion harmonics; it uses real-time digital signal storage and phase cancellation techniques to create a harmonic signal. "SonoCT" is ATL-Philips' proprietary name for spatial compound imaging; it works by averaging between three (survey mode) and nine (target mode) frames taken with beams orientated at different angles within the same imaging field. HSonoCT is an imaging technique which uses a combination of SonoCT imaging with THI. In this study, nine frames were used throughout to optimise the image quality of SonoCT and HSonoCT.

\section{Tissue Mimicking Phantom}

The L7-4 and L12-5 image quality measurements were performed using the Gammex-RMI model 404GS LE small parts test object, whereas the C5-2 image quality measurements were performed using the Gammex-RMI model 403GS LE general purpose test object. The tissue-mimicking material used in both phantoms was an evaporated-milk-based gel; its acoustic properties are listed in Table 1. 


\section{Image Capture and Computerised Analysis}

In order to evaluate the image quality of the three probes using the two test objects, the following procedure was used. The appropriate target within the test object was imaged firstly using conventional B-mode. The image was optimised and captured using the systems own MO disk facility for off-line analysis using a computerised quality control testing system developed by the Medical Physics Dept., Nottingham City Hospital (Gibson et al 2001). The next imaging mode was then activated, the image was optimised again and captured. The C5-2 probe was tested with the "Abdominal-Abdo" preset, the L7-4 probe was tested with the "General-Generic" preset and the L12-5 was tested with the "Small Parts-Thyroid" preset. The presets chosen for the C5-2 and the L12-5 probes were those which have been optimised for imaging the abdomen and the thyroid, respectively, and normally used clinically. The L7-4 probe was normally used clinically to image the femoral artery; however, the preset used in this study was General Generic, in order for the THI mode to be available. There were three optimisation settings available for conventional B-mode and THI: "Res" (optimisation in the near field of the image); "Gen" (optimisation over the entire image field of view); and "Pen" (optimisation in the far field of the image). The optimisation setting used for the conventional B-mode of all three probes was "Gen". The optimisation mode for C5-2 THI mode was also "Gen"; however, the "Res" optimisation setting was used for the L7-4 THI mode, as this was the only setting available. SonoCT and harmonic SonoCT (HSonoCT) had two modes, "Survey" for target location with reasonable frame rate performance and "Target" for optimal image quality. The latter mode was used for testing both SonoCT and HSonoCT. The optimisation procedure for all modes involved adjusting the 2-D gain and the time-gain-controls, as well as choosing the appropriate number of focal zones 
to give the best possible image of the test object target under test. The position and number of focal points were kept constant between modes. The acoustic power settings used for the C5-2, the L7-4 and the L12-5 probes were MI $=1.3, \mathrm{MI}=0.6$ and $\mathrm{MI}=0.42$, respectively. The MI and TIS values for the three different probes changed automatically with each of the modes; in general, the MI values for the THI mode, SonoCT mode and the HSonoCT mode were greater than the MI value for the conventional B-mode. The probe was held in place throughout the test period of each imaging mode using a clamp and retort stand. This process was repeated for each set of the targets within the test object for the three probes.

Axial and Lateral Resolution, and Slice Thickness. Axial and lateral resolution and slice thickness were measured by the automated image analysis program by drawing a region-of-interest around the cross-sectional image of the nylon filament targets and measuring the full-width half-maximum (FWHM) above the surrounding background of the filament in both the axial and lateral direction. Slice thickness was assessed using the Skolnick method (Skolnick, 1991). The vertical column of filaments was imaged throughout the useful field of view; the probe was rotated through $45^{\circ}$ and held against an alignment marker to ensure accuracy. The uncertainty $(95 \%$ confidence interval $(\mathrm{CI}))$ in the measurement of these three parameters due to reanalysis using the computerised quality control testing system (USQA) was $\pm 1.2 \%$ (axial resolution) and $\pm 1.5 \%$ (lateral resolution and slice thickness); this represents the uncertainty in comparison between different imaging modes with the probe clamped. 
Contrast Resolution. Contrast resolution was measured using the automated image analysis program, by selecting points at opposite positions of the contrast target's circumference. The program then drew two circles, one within the target and the other outside the target. Contrast resolution was expressed as a visibility index of each of the targets, by calculating the ratio of the difference of means between the target $\left(M_{\text {in }}\right)$ and the background $\left(\mathrm{M}_{\text {out }}\right)$ area and the standard error (SE) between the two means (eqn 1).

$$
\text { Visibility Index }=\left[\mathrm{M}_{\mathrm{in}}-\mathrm{M}_{\text {out }}\right] /[\mathrm{SE}]
$$

The uncertainty $(95 \% \mathrm{CI})$ in the visibility index measurement due to reanalysis using the USQA program was $\pm 3 \%$; this represents the uncertainty in comparison between different imaging modes with the probe clamped.

Anechoic Target Detection Ability. Anechoic target detection was determined by the automated image analysis program by drawing a region-of-interest around the array of anechoic targets and calculating the correlation coefficient between the detected target and an ideal target using a matched filter. The correlation coefficient $\mathrm{R}$ was then transformed into $\mathrm{Z}$ according to eqn 2 . $\mathrm{Z}$ is normally distributed, enabling the above calculation of confidence interval from its standard deviation (Altman 1991).

$$
\mathrm{Z}=1 / 2 \ln [(1+\mathrm{R}) /(1-\mathrm{R})]
$$

The uncertainty $(95 \% \mathrm{CI})$ in the anechoic target detection measurement due to reanalysis using the USQA program was $\pm 1 \%$; this represents the uncertainty in comparison between different imaging modes with the probe clamped. 
Low Contrast Penetration Depth. Low contrast penetration depth was measured by the automated image analysis program by capturing two images in quick succession, the only difference between them being the electronic noise present. A region-of-interest was drawn around an area free from targets and the low contrast penetration depth was determined as the point at which the ratio between speckle and noise (SNR) fell below a specified threshold value of 2.5 , determined by the minimum signal-to-noise ratio of 2.5 to 5 necessary to distinguish weak echoes from noise (Gibson et al 2001). The uncertainty (95\% CI) in the low contrast penetration depth measurement due to reanalysis using the USQA program was $\pm 4 \%$; this represents the uncertainty in comparison between different imaging modes with the probe clamped.

\section{RESULTS}

The performance of conventional B-mode, THI, SonoCT and HSonoCT imaging modes for three probes of the HDI 5000 were evaluated objectively using a computerised quality control testing system. The performance results for the different imaging modes for the three probes, C5-2, L7-4 and L12-5 of the HDI 5000 are presented in Figs. 1 to 13

\section{Axial Resolution}

The axial resolution of the C5-2, L7-4 and L12-5 probes as a function of depth in either the general purpose (model 403LE GS) or the small parts (model 404LE GS) test objects can be seen in Figs. 1 to 3. 
THI Mode. A modest improvement $(\mathrm{p}<0.05)$ in axial resolution was observed for the C5-2 probe with THI (Fig. 1). The L7-4 probe had a more significant improvement $(\mathrm{p}<0.05)$ in axial resolution with THI after a depth of $30 \mathrm{~mm}$.

SonoCT Mode. The SonoCT mode for all three probes were found to be similar to that of the conventional B-mode.

HSonoCT Mode. HSonoCT mode was found to have similar axial resolution to that of conventional B-mode for the C5-2 probe while, for the L7-4 probe, the axial resolution with the HSonoCT mode was worse $(\mathrm{p}<0.05)$ than that of conventional B-mode up to a depth of $40 \mathrm{~mm}$, at which point it improved and was better than that of conventional B-mode (Figs. 1 and 2).

\section{Lateral Resolution}

The lateral resolution of the C5-2, L7-4 and L12-5 probes as a function of depth in either the general purpose (model 403LE GS) or the small parts (model 404LE GS) test objects can be seen in Figs. 4 to 6 .

THI Mode. A significant improvement $(\mathrm{p}<0.05)$ in lateral resolution was observed for the C5-2 probe with THI (Fig. 4). The L7-4 probe also had a significant improvement $(\mathrm{p}<0.05)$ in lateral resolution after a depth of $30 \mathrm{~mm}$ with THI.

SonoCT Mode. For all three probes the lateral resolution of SonoCT mode was worse $(p<0.05)$ than that of conventional B-mode (Figs. 4 to 6$)$.

HSonoCT Mode. Modest improvements $(\mathrm{p}<0.05)$ in lateral resolution compared with conventional B-mode for the C5-2 and the L7-4 probes were observed with HSonoCT after $100 \mathrm{~mm}$ and $45 \mathrm{~mm}$ respectively (Figs. 4 and 5).

Slice Thickness 
The average slice thickness of the C5-2, L7-4 and L12-5 probes as a function of depth in either the general purpose (model 403LE GS) or the small parts (model 404LE GS) test objects can be seen in Figs. 7 to 9 .

THI Mode. Significant improvements $(\mathrm{p}<0.05)$ for the C5-2 probe and the L7-4 probes were observed in the slice thickness with THI.

SonoCT Mode. The slice thickness of the SonoCT modes for all three probes were worse $(\mathrm{p}<0.05)$ than that of the conventional B-mode.

HSonoCT Mode. Significant improvements $(\mathrm{p}<0.05)$ in slice thickness were observed with HSonoCT for the C5-2 and the L7-4 probes (Figs. 7 and 8).

\section{Contrast resolution}

The contrast resolution results of the C5-2, L7-4 and L12-5 probes for the different grey-scale targets in either the general purpose (model 403LE GS) or the small parts (model 404LE GS) test objects can be seen in Figs. 10 to 12.

THI Mode. The contrast resolution visibility indices for the C5-2 and L7-4 THI modes were worse $(\mathrm{p}<0.05)$ than that of the conventional B-mode visibility indices (Figs. 10 to 13 ).

SonoCT Mode. For all three probes, the contrast resolution visibility indices of SonoCT mode were better $(\mathrm{p}<0.05)$ than that of conventional B-mode (Figs. 10 to 12).

HSonoCT Mode. For the C5-2 and L7-4 probes, the contrast resolution visibility indices of the HSonoCT mode was better $(p<0.05)$ than that of the conventional B-mode visibility indices (Figs. 10 and 11). 
Anechoic Target Detection

The anechoic target detection results of the C5-2, L7-4 and L12-5 probes for the anechoic targets as a function of depth in either the general purpose (model 403LE GS) or the small parts (model 404LE GS) test objects can be seen in Tables 2 to 4 .

THI Mode. For the C5-2 and L7-4 probes, the ability to detect the anechoic targets of variable sizes and at increasing depths was in general worse $(\mathrm{p}<0.05)$ with the THI mode than that of the conventional B-mode (Tables 2 and 3).

SonoCT Mode. For all three probes, the ability to detect the anechoic targets of variable sizes and at increasing depths was in general better $(\mathrm{p}<0.05)$ with the SonoCT mode than that of the conventional B-mode (Tables 2 to 4).

HSonoCT Mode. For the C5-2 and L7-4 probes, the ability to detect the anechoic targets of variable sizes and at increasing depths was either similar to or better $(\mathrm{p}<0.05)$ than that of the conventional B-mode (Tables 2 and 3$).$

\section{Low Contrast Penetration Depth}

The low contrast penetration depth results of the C5-2, L7-4 and L12-5 probes in either the general purpose (model 403LE GS) or the small parts (model 404LE GS) test objects can be seen in Fig. 13.

THI Mode. The low contrast penetration depth with THI mode for both the C5-2 and L7-4 probes was found to be worse $(p<0.05)$ than that of the conventional B-mode (Fig. 13).

SonoCT Mode. The low contrast penetration depth with the SonoCT mode was found to be similar or slightly better $(\mathrm{p}<0.05)$ than that of the conventional B-mode (Fig. 13). 
HSonoCT Mode. The low contrast penetration depth with HSonoCT mode for both the C5-2 and L7-4 probes was found to be worse $(\mathrm{p}<0.05)$ than that of the conventional B-mode (Fig. 13).

\section{DISCUSSION}

The performance of conventional B-mode, THI, SonoCT and HSonoCT imaging modes of the C5-2, L7-4 and L12-5 probes of the HDI 5000 ultrasound scanner were objectively evaluated, using a computerised quality control testing system. THI improved the overall spatial resolution of the C5-2 and L7-4 probes compared with the conventional B-mode. SonoCT greatly improved the contrast resolution and the anechoic target detection of the three probes tested. HSonoCT improved the overall spatial resolution and the anechoic target detection of the C5-2 and L7-4 probes. The improvements in axial resolution with THI mode compared with the conventional B-mode is likely to be due to the higher frequency of the returning harmonic pulse or the shorter pulse length. The improvements which were found for lateral resolution and slice thickness for THI and HSonoCT can be attributed to reduced second harmonic beam width (Ward et al 1997). The improvements in lateral resolution and slice thickness for the L7-4 probe with THI were only present after a depth of $40 \mathrm{~mm}$, which is most likely the point at which harmonic generation became significant. The improvements found in lateral resolution and slice thickness are consistent with the improvements in overall image quality which have been reported in clinical studies. Limited improvements in spatial resolution with SonoCT mode compared with the conventional B-mode were found for the C5-2, L7-4 or L12-5 probes. These results were expected, since neither the pulse length nor beam width are altered by using SonoCT; however, for the C5-2 probe at depth where the scan line density is 
decreased and interpolation of the scan lines is needed, the compounded image should demonstrate an improvement in lateral resolution.

Tissue harmonic imaging did not improve contrast resolution or anechoic target detection compared with the conventional B-mode for either the C5-2 or L7-4 probes. These results do not correlate with the reported improvements in contrast resolution and lesion detection across a wide range of clinical applications (Puls et al 2000; Tanaka et al 2000; Tranquart et al 1999; Shapiro et al 1998). In particular, THI has been reported as having improved anechoic lesion detectability and low contrast lesion detectability obtainable from patients who are difficult to image with conventional B-mode (Desser and Jeffrey 2001). The absence of improvements in contrast resolution and anechoic target detection with THI may be due to the choice of the test objects used in this study. The test objects used were of simple homogeneous design with no fat-mimicking layers, therefore, causing no phase aberration or distortion in the beam as would be found in-vivo. THI improves the clinical image quality in-vivo by passing only once through the body wall and the fat layers, thus reducing the effects of phase aberrations. A future study evaluating the image quality performance of THI should include the use of a test object that simulates the body wall and fat layers. Furthermore, the tissue harmonic images from the C5-2 and L7-4 probes appeared to be darker and noisier than the conventional B-mode image, thereby reducing the ability to detect anechoic targets (Figs. 14 and 15). This may be a result of the harmonic signal being at least $20 \mathrm{~dB}$ lower than the fundamental signal, resulting in a lower dynamic range for the harmonic signal (Desser et al 2000). The increased noise recorded by the system could be due to the need for increased receiver sensitivity to 
pick up low amplitude harmonic signals, as well as the need to widen the bandwidth (Ward et al 1997; Desser et al 2000).

SonoCT was found to improve substantially both contrast resolution and anechoic target detection for the three probes tested. The improvement in contrast resolution and anechoic detection with SonoCT is based on averaging images of the same object but acquired from different view angles (Entrekin et al 2000). If $\mathrm{N}$ frames are acquired from sufficiently different view angles, the frame-to-frame speckle patterns will be uncorrelated and averaging these frames will increase the signal-to-noise ratio (SNR) by a factor of $\sqrt{ } \mathrm{N}$ and, thus, the detectability of a variable contrast or anechoic target. The detectability of variable contrast or anechoic target is a function of spatial resolution, statistical properties of speckle pattern in the target and background, as well as the target contrast and size. The effect of spatial compounding on the variable contrast or anechoic target detectability should also be increased by a factor of $\sqrt{ } \mathrm{N}$ (where $\mathrm{N}=9$ ), therefore increasing the visibility index of contrast targets and the $\mathrm{Z}$ coefficient of anechoic targets. However, the transmit and receive apertures of the C52, L7-4 and the L12-5 probes of the HDI 5000 are reported as being the same for both conventional B-mode and SonoCT, therefore the actual gain in speckle reduction is modest compared with the theoretical estimates (Entrekin et al 2000).

The low contrast penetration depth decreased with THI and HSonoCT compared with the conventional B-mode for the C5-2 and L7-4 probes; these results were as expected and due to the lower amplitude of the received second harmonic. SonoCT had similar low contrast penetration to the conventional B-mode for all three probes. 


\section{Conclusions}

In this paper, differences between the performance of conventional B-mode, THI, SonoCT and HSonoCT imaging modes of the HDI 5000 were objectively evaluated using a computerised quality control testing system. The measurement limitations associated with subjective analysis methods were not present in this study, since an automated image analysis program was used to determine the image quality parameters; therefore, subtle differences between the four imaging modes could be detected. It was found that THI improved the spatial resolution of the C5-2 and L7-4 probes, in particular, lateral resolution and slice thickness. No improvements in contrast resolution or anechoic target detection were observed with the THI mode compared with that of the conventional B-mode for the C5-2 and L7-4 probes; further work needs to be carried out to determine whether the use of fat layers will result in improved contrast resolution or anechoic detection with the use of THI. THI was found to cause a decrease in penetration depth of both of these probes tested. SonoCT was found to improve the contrast resolution and the anechoic target detection of the C5-2, L7-4 and L12-5 probes. HSonoCT was found to improve the spatial resolution, contrast resolution and anechoic target detection of the C5-2 and L 7-4 probes, a combination of the improvements found for THI mode and SonoCT. 


\section{REFERENCES}

Averkiou MA, Roundhill DN and Powers JE. A new imaging technique based on the nonlinear properties of tissues. IEEE Ultrasonics Symp 1997; 1561 - 1566.

Christopher T. Finite amplitude distortion-based inhomogeneous pulse echo ultrasonic imaging. IEEE Trans Ultras Ferro Freq Control 1997; 44: 125 - 139.

Claudon M, Tranquart F, Evans DH, Lefevre F and Correas JM. Advances in ultrasound. Europ Radiol. 2002; 12: 7-18.

Desser TS, Jedrzejewicz T and Bradley C. Native tissue harmonic imaging: Basic principles and clinical applications. Ultrasound Quart 2000; 16: 40 - 48.

Desser TS and RB Jeffrey. Tissue harmonic imaging techniques: Physical principles and clinical applications. Semin Ultrasound, CT and MRI 2001; 22: 1 - 10.

Dudley NJ, Gibson NM, Fleckney MJ and Clarke PD. The effect of speed of sound in ultrasound test objects on lateral resolution. Ultrasound Med Biol 2002; 28: 1561 1564.

Entrekin RR, Jago JR and Kofoed SC. Real-time spatial compound imaging: technical performance in vascular applications. Acoustical Imaging 2000; 25: 331 - 342. 
Entrekin RR, Porter BA, Sillesen HH, Wong AD, Cooperberg PL and Fix CH. Realtime spatial compound imaging: application to breast, vascular and musculoskeletal ultrasound. Semin Ultrasound, CT and MRI 2001; 22: $50-64$.

Gatenby JC, Hoddinott JC and Leeman S. Phasing out speckle. Phys Med Biol 1989; 34: $1683-1689$.

Gibson NM, Dudley NJ and Griffith K. A computerised quality control testing system for B-mode ultrasound. Ultrasound Med Biol 2001;27: 1697 - 1711.

Goldstein A. The effect of speed of sound on phantom measurements. Ultrasound Med Biol 2000;26: 1133 - 1143.

Goodsitt MM, Carson PL, Witt S, Hykes DL, Kofler JM. Real-time B-mode ultrasound quality control test procedures. Report of AAPM Ultrasound Task Group No. 1. Med Phys 1998; 25: 1385-1406.

Howry DH. Techniques used in ultrasound visualization of soft tissue structures of the body. IRE Conv Rec 1955; 9: 75 - 81 .

Jespersen SK, Wilhjelm JE and Sillesen H. In vitro spatial compound scanning for improved visualization of atherosclerosis. Ultrasound Med Biol 2000; 26: 1357 1362. 
Li Y and Zagzebski JA. Computer model for harmonic ultrasound imaging. IEEE Trans Ultras Ferro Freq Control 2000; 47: 1000 - 1013.

Price R, editor. Routine quality assurance of ultrasound imaging systems. York: The Institute of Physical Sciences in Medicine, 1995.

Puls I, Berg D, Maurer M, Schliesser M, Hetzel G and Becker G. Transcranial sonography of the brain parenchyma: comparison of B-mode imaging and tissue harmonic imaging. Ultrasound Med Biol 2000; 26: 189 - 194.

Shapiro RS, Wagreich J, Parsons RB, Stancato-Pasik A, Yeh H and Lao R. Tissue harmonic imaging sonography: evaluation of image quality compared with conventional sonography. Am J Roentgen 1998; 171: 1203 - 1206.

Skolnick ML. Estimation of ultrasound beam width in the elevation (section thickness) plane. Radiology 1991; 180: 286 - 288.

Tanaka S, Oshikawa O, Sasaki T, Ioka T and Tsukuma H. Evaluation of tissue harmonic imaging for the diagnosis of focal liver lesions. Ultrasound Med Biol 2000; 26: $183-187$.

Tranquart F, Grenier N, Eder V and Pourcelot L. Clinical use of ultrasound tissue harmonic imaging. Ultrasound Med Biol 1999; 25: 889 - 894. 
Ward B, Backer AC and Humphrey VF. Nonlinear propagation applied to the improvement of resolution in diagnostic medical ultrasound. J Acoust Soc Am 1997; 101: $143-154$. 
Table 1. Reported Acoustic Data of the 403LE GS and 404LE GS test phantom tissue-mimicking material.

\begin{tabular}{c||c|c|c}
\hline \hline $\begin{array}{c}\text { Tissue-mimicking } \\
\text { material }\end{array}$ & $\begin{array}{c}\text { Speed of sound } \\
(\mathbf{m} / \mathbf{s})\end{array}$ & $\begin{array}{c}\text { Attenuation coefficient } \\
(\mathbf{d B} / \mathbf{c m M H z})\end{array}$ & $\begin{array}{c}\text { Non-linearity } \\
\text { parameter }\end{array}$ \\
\hline \hline Condensed milk gel & 1540 & 0.7 & 6.6 \\
$($ Model 403GS LE) & $@ 2-18 \mathrm{MHz}$ & $@ 2-18 \mathrm{MHz}$ & \\
$($ Model 404GS LE) & & & \\
\hline \hline
\end{tabular}

Table 2. Z-coefficient of three different anechoic targets (cyst size: $6 \mathrm{~mm}, 4 \mathrm{~mm}$ and $2 \mathrm{~mm}$ ) as a function of depth for the four modes of the C5-2 probe in the general purpose test object. (Focal points at 30,70110 and $180 \mathrm{~mm}$.)

\begin{tabular}{cccccc}
\hline \hline Cyst size - Depth & B-mode & THI mode & SonoCT & HSonoCT \\
\hline $\mathbf{m m})$ & $(\mathbf{m m})$ & & mode & mode \\
\hline \hline 6 & 30 & 1.31 & 1.14 & 1.33 & 1.32 \\
6 & 80 & 1.19 & 1.09 & 1.23 & 1.24 \\
6 & 140 & - & - & - & - \\
4 & 30 & 1.26 & 1.04 & 1.33 & 1.19 \\
4 & 80 & 1.22 & 1.12 & 1.33 & 1.29 \\
4 & 140 & - & - & - & - \\
2 & 30 & 0.81 & 0.71 & 0.7 & 1.07 \\
2 & 80 & 0.74 & - & 0.85 & 0.77 \\
2 & 140 & - & - & - & - \\
\hline \hline
\end{tabular}


Table 3. Z-coefficient of three different anechoic targets (cyst size: $4 \mathrm{~mm}, 2 \mathrm{~mm}$ and $1 \mathrm{~mm}$ ) as a function of depth for the four modes of the $\mathbf{~ 7 - 4}$ probe in the small parts test object. (Focal points at 35, 4555 and $70 \mathrm{~mm}$.)

\begin{tabular}{cccccc}
\hline $\begin{array}{c}\text { Cyst size } \\
(\mathbf{m m})\end{array}$ & $\begin{array}{c}\text { Depth } \\
(\mathbf{m m})\end{array}$ & B-mode & THI mode & SonoCT & HSonoCT \\
\hline \hline 4 & 10 & 1.01 & 0.89 & 1.1 & mode \\
4 & 35 & 1.08 & 0.95 & 1.19 & 1.32 \\
4 & 60 & 0.57 & - & - & - \\
2 & 10 & 0.91 & 0.95 & 1.18 & 1.24 \\
2 & 35 & 0.89 & 0.92 & 0.98 & 0.99 \\
2 & 60 & 0.44 & - & - & - \\
1 & 10 & 0.81 & 0.75 & 1.03 & 1.07 \\
1 & 35 & 0.68 & 0.68 & 0.79 & 0.86 \\
1 & 60 & 0.57 & - & - & - \\
\hline \hline
\end{tabular}


Table 4. Z-coefficient of three different anechoic target (cyst size: $4 \mathrm{~mm}, 2 \mathrm{~mm}$ and $1 \mathrm{~mm}$ ) as a function of depth for the two modes of the L12-5 probe in the small parts test object. (Focal points at 30, 40 and $60 \mathrm{~mm}$.)

\begin{tabular}{cccc}
\hline $\begin{array}{c}\text { Cyst size } \\
(\mathbf{m m})\end{array}$ & $\begin{array}{c}\text { Depth } \\
(\mathbf{m m})\end{array}$ & B-mode & SonoCT \\
\hline 4 & 10 & 1.09 & 1.33 \\
4 & 35 & 1.05 & 1.12 \\
4 & 60 & - & - \\
2 & 10 & 1.08 & 1.25 \\
2 & 35 & 1.04 & 1.24 \\
2 & 60 & - & - \\
1 & 10 & 0.79 & 1.16 \\
1 & 35 & 0.75 & 0.92 \\
1 & 60 & - & - \\
\hline \hline
\end{tabular}


Fig. 1. Axial resolution as a function of depth of the C5-2 probe four different modes in the Model 403GS LE test object. (Focal points @ 30, 70110 and 180mm)

Fig. 2. Axial resolution as a function of depth of the L7-4 probe four different modes in the Model 404GS LE test object. (Focal points @ 35, 4555 and $70 \mathrm{~mm}$ )

Fig. 3. Axial resolution as a function of depth of the L12-5 probe two different modes in the Model 404LE test object. (Focal points @ 30, 40 and 60 mm)

Fig. 4. Lateral resolution as a function of depth of the $\mathrm{C} 5-2$ probe four different modes in the Model 403LE test object. (Focal points @30,70110 and 180mm)

Fig.5. Lateral resolution as a function of depth of the L7-4 probe four different modes in the Model 404LE test object. (Focal points@35, 4555 and 70 mm)

Fig. 6. Lateral resolution as a function of depth of the L12-5 probe two different modes in the Model 404LE test object. (Focal points @ 30, 40 and 60 mm)

Fig. 7. Slice thickness as a function of depth of the C5-2 probe four different modes in the Model 403LE test object. (Focal points@30,70110 and 180mm)

Figure 8. Slice thickness as a function of depth of the L7-4 probe four different modes in the Model 404LE test object. (Focal points@35, 4555 and 70 mm)

Fig. 9. Slice thickness as a function of depth of the L12-5 probe two different modes in the Model 404LE test object. (Focal points @ 30, 40 and 60 mm)

Fig. 10. Contrast Resolution of the C5-2 probe four different modes for the Model 403GS LE test object. (Focus @ 60mm)

Fig. 11. Contrast Resolution of the L7-4 probe four different modes for the Model 404GS LE test object. (Focus @ 29mm)

Fig. 12. Contrast Resolution of the L12-5 probe two different modes for the Model 404GS LE test object. (Focus @ 29mm)

Fig. 13. Low Contrast Penetration Depth Results of the C5-2, L7-4 and L12-5 probe four different modes in the Model 403GS LE and Model 404GS LE test objects.

Fig.14. L7-4 B-mode image of the $-12 \mathrm{~dB}$ and $-6 \mathrm{~dB}$ contrast targets in the Model 404GS LE test object.

Fig. 15. L7-4 THI image of the $-12 \mathrm{~dB}$ and $-6 \mathrm{~dB}$ contrast targets in the Model 404GS LE test object. 


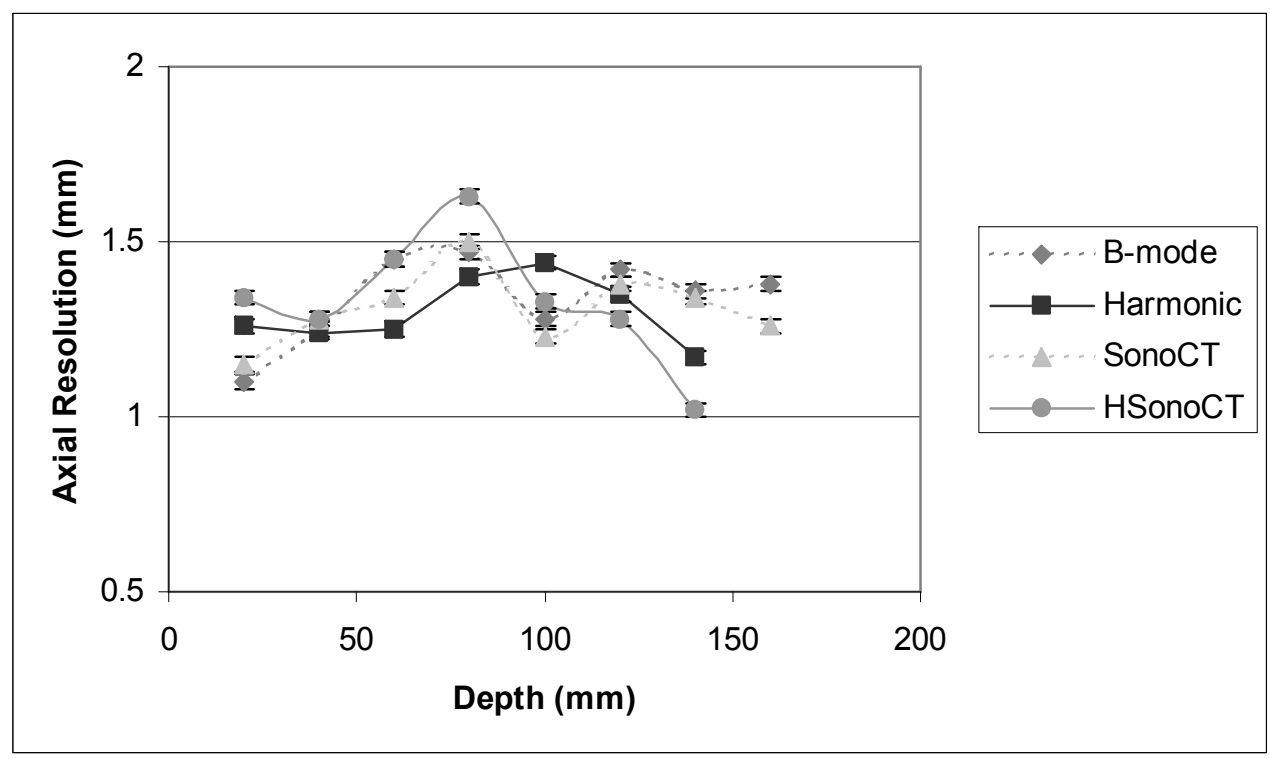

Fig. 1. 


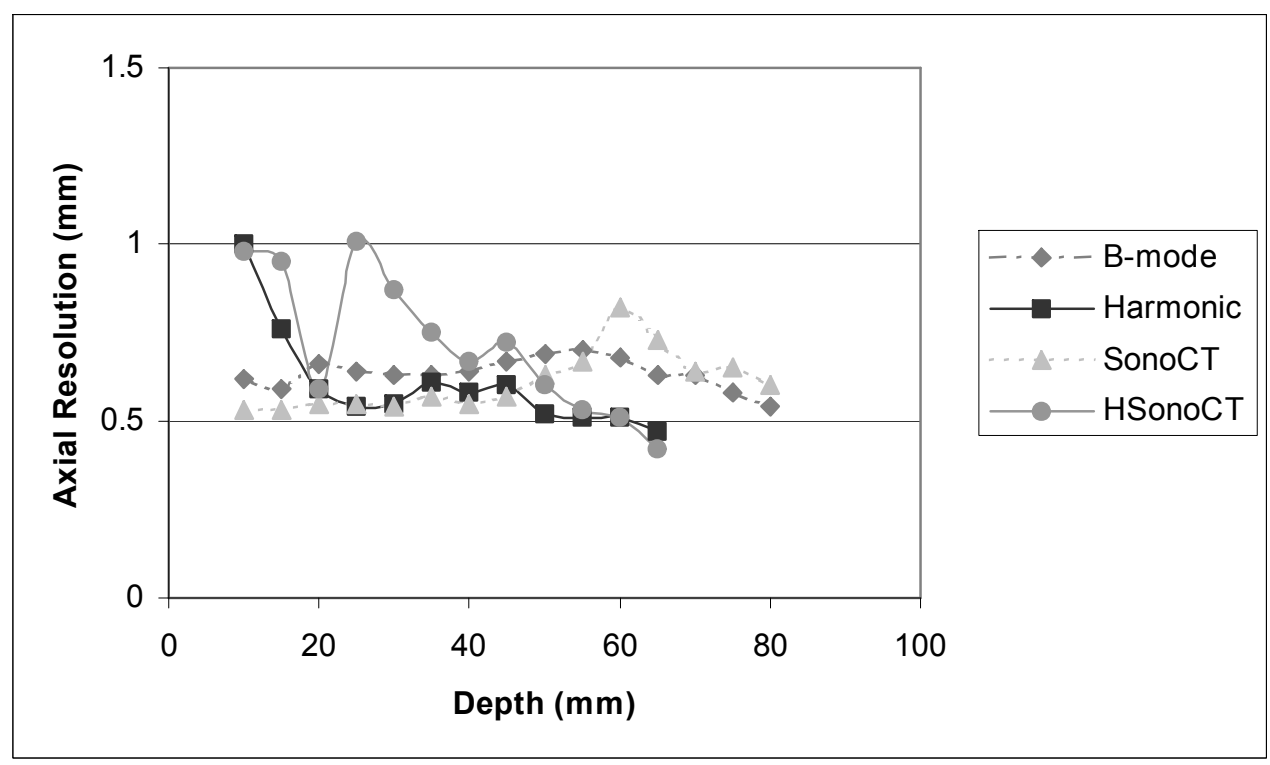

Fig.2. 


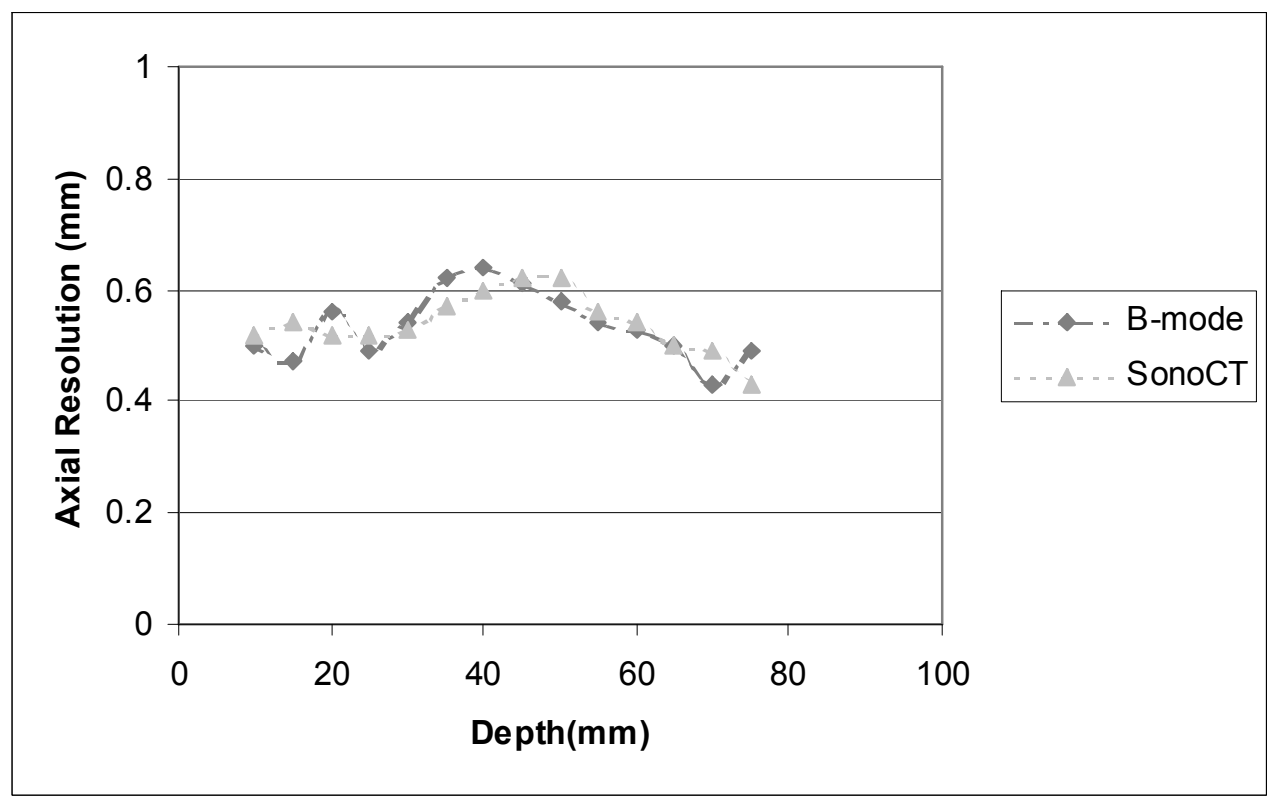

Fig. 3. 


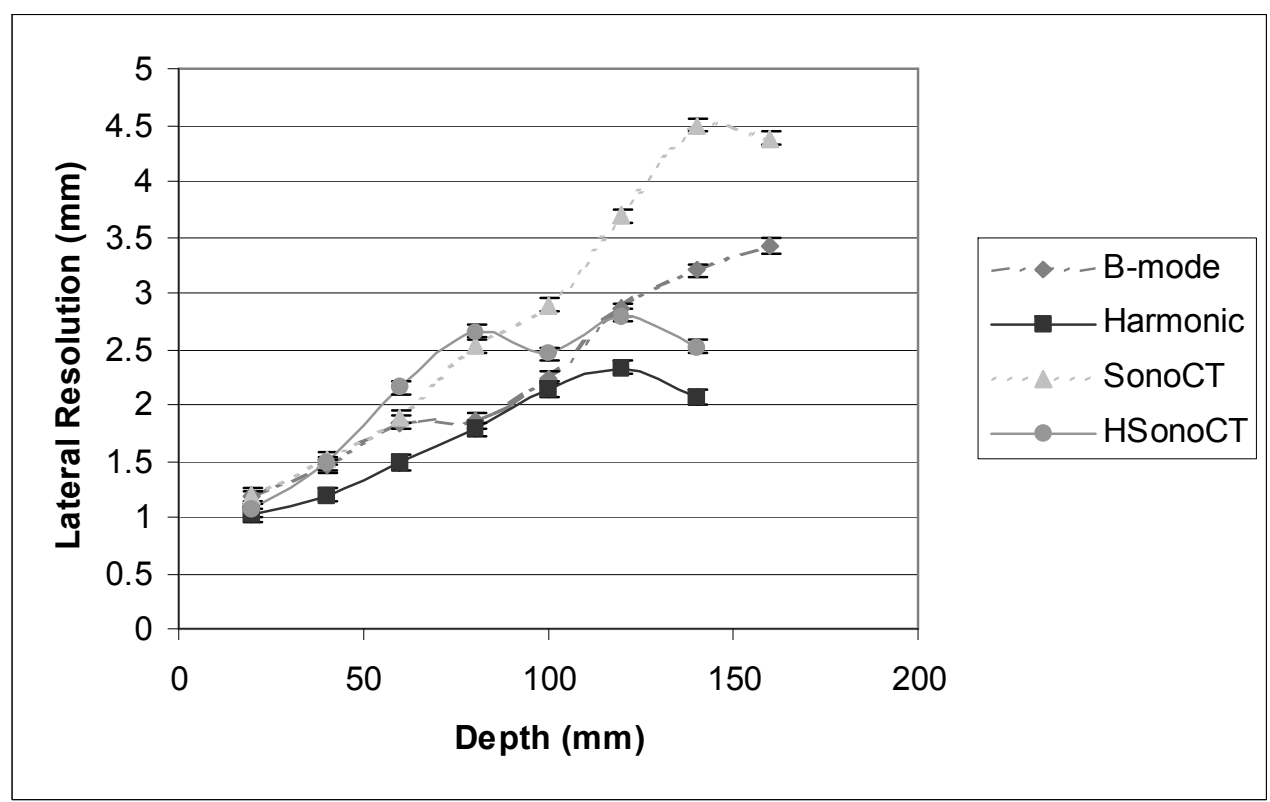

Fig. 4. 


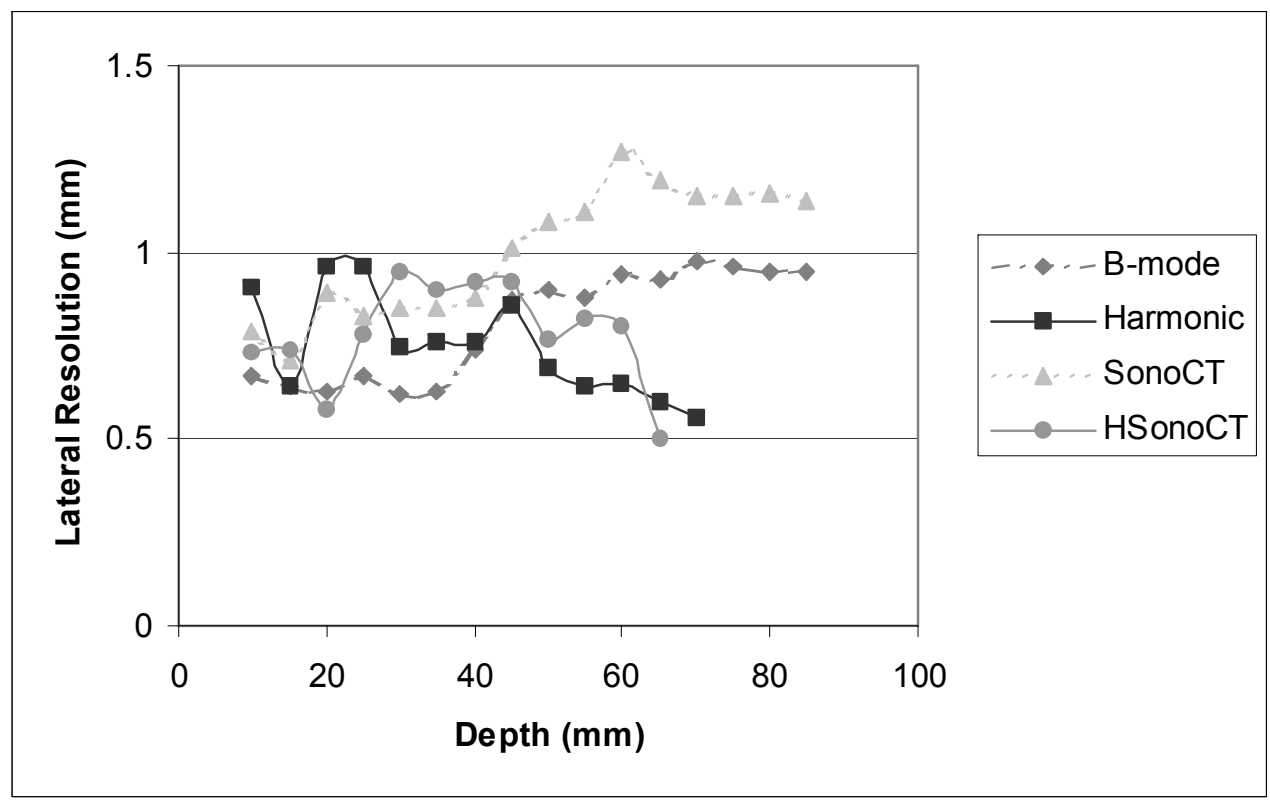

Fig. 5. 


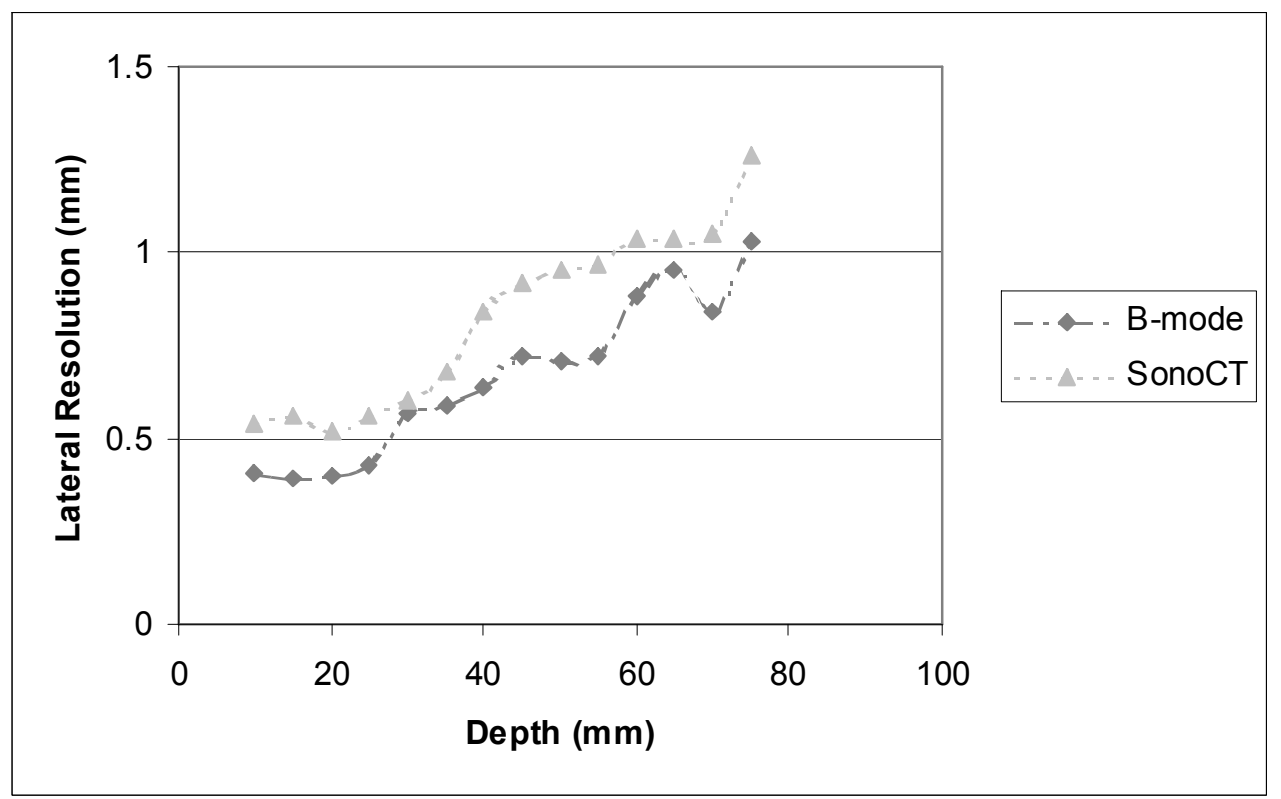

Fig. 6. 


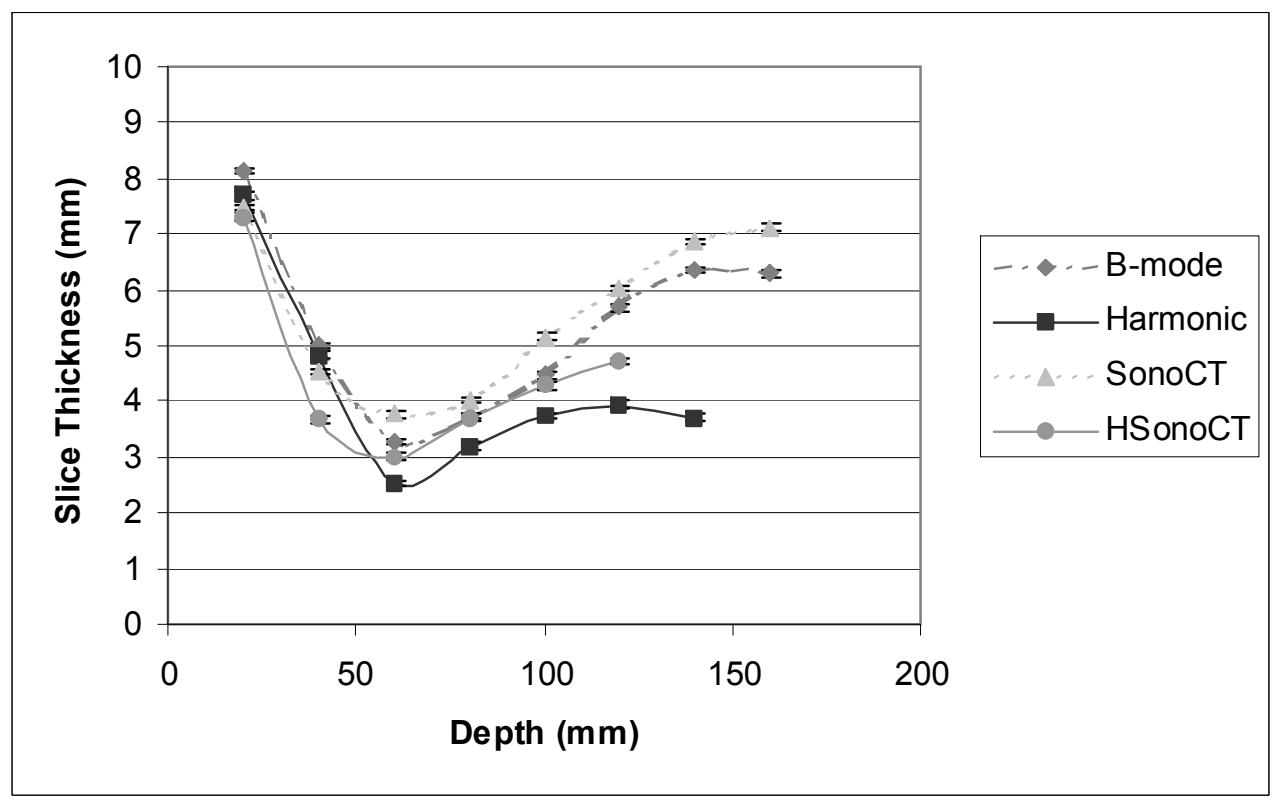

Fig. 7. 


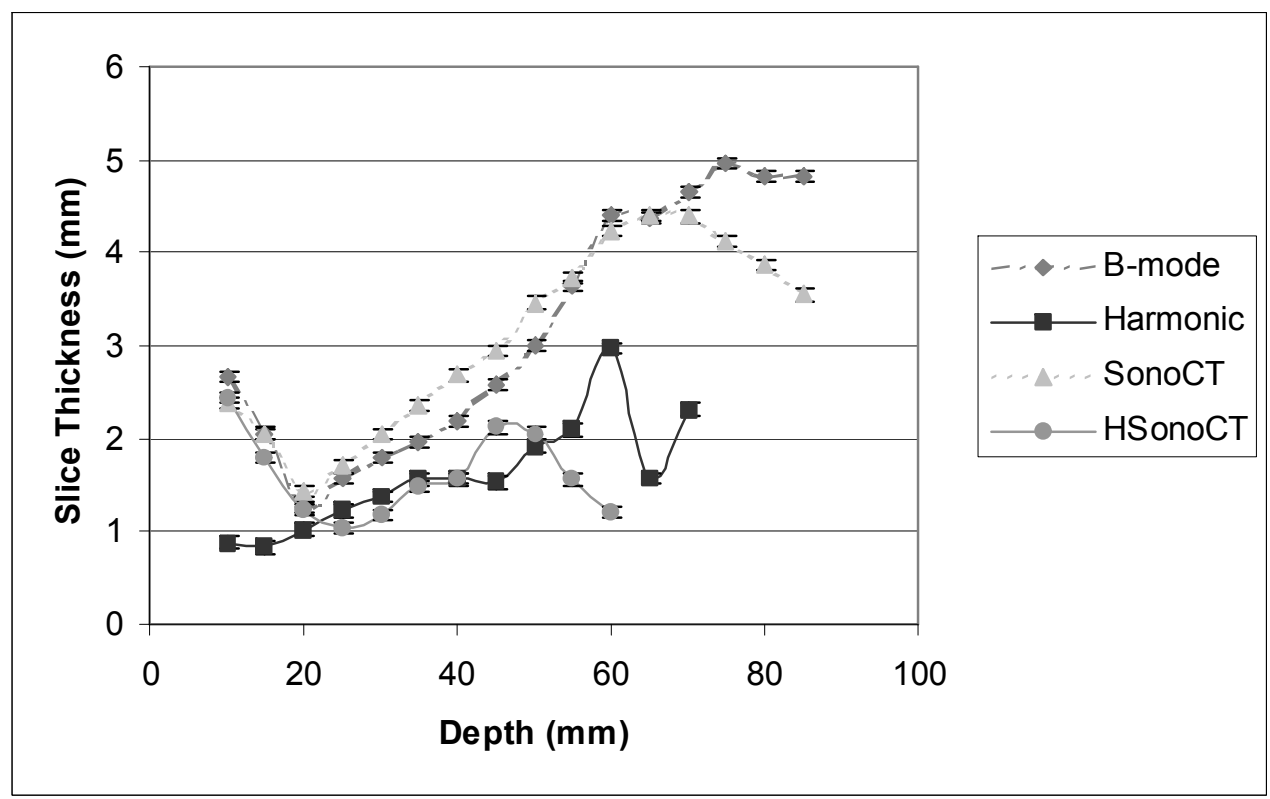

Fig. 8 . 


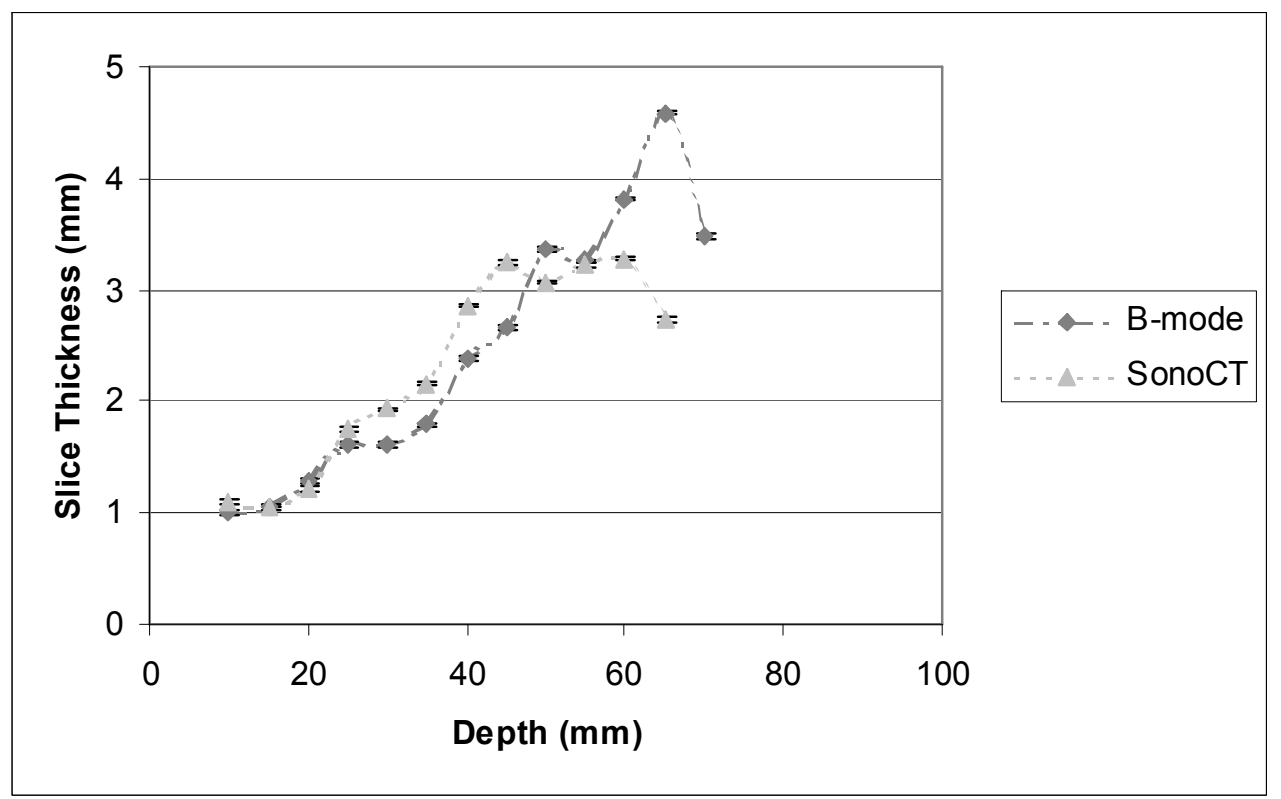

Fig. 9. 


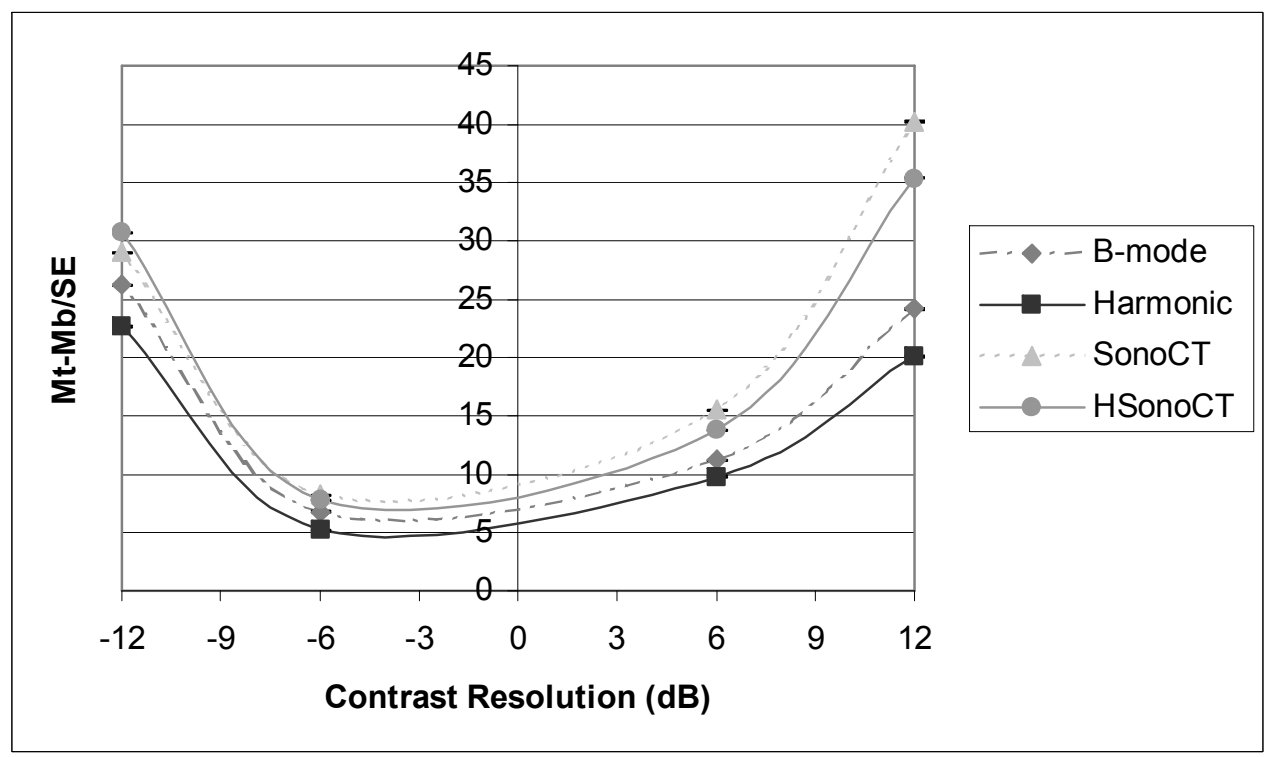

Fig. 10 . 


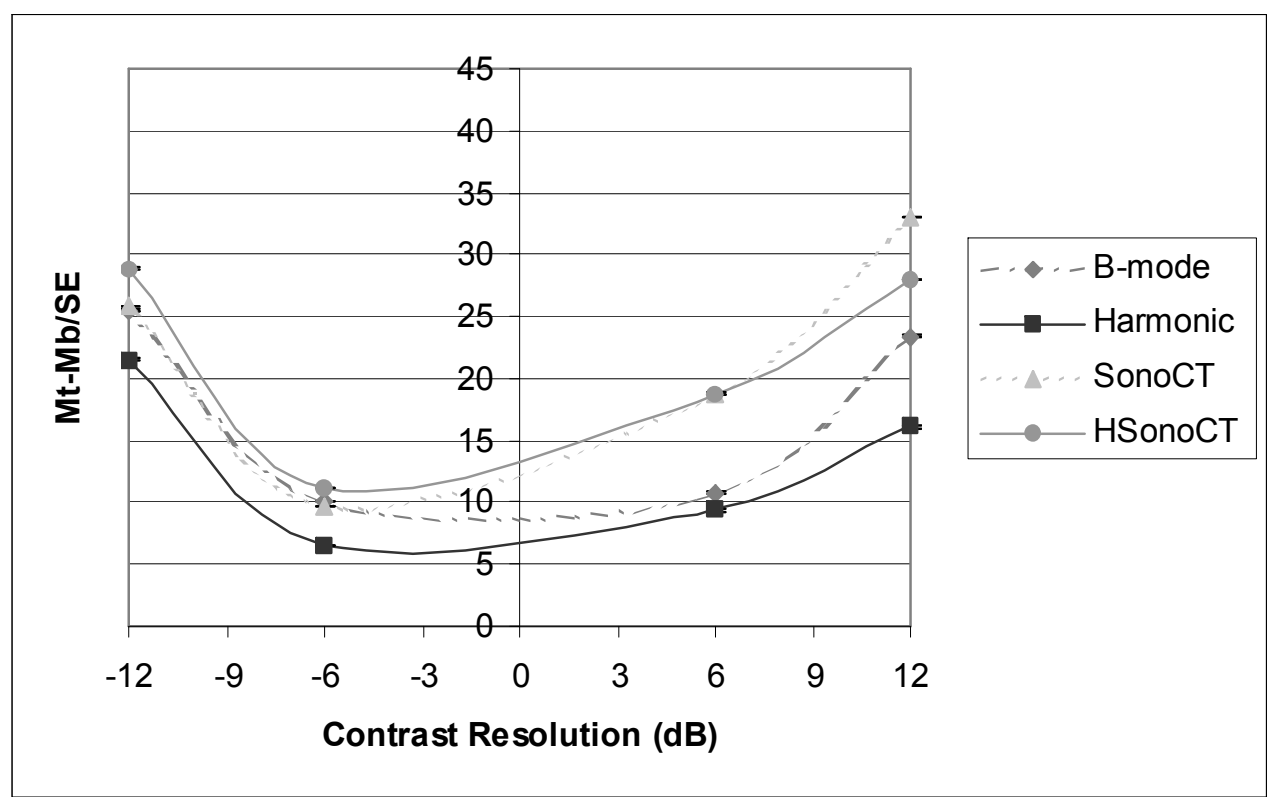

Fig. 11. 


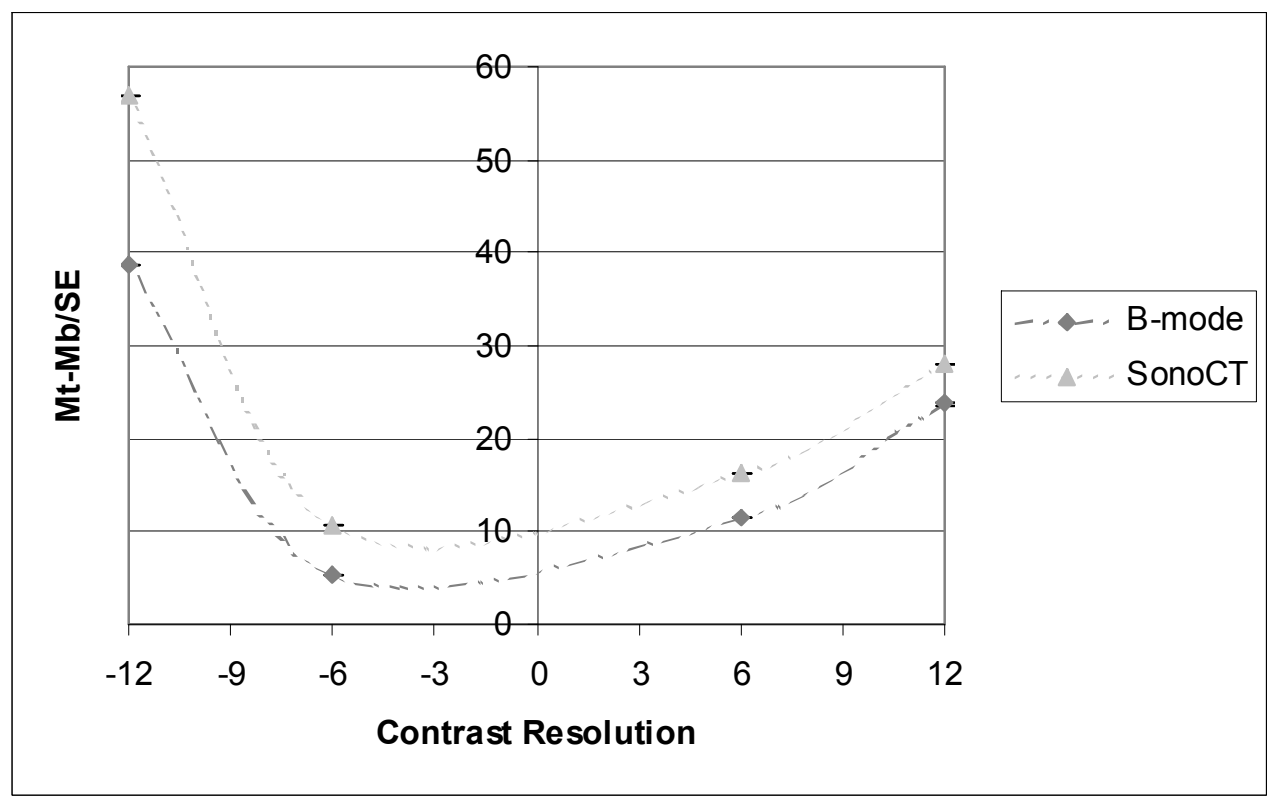

Fig. 12. 


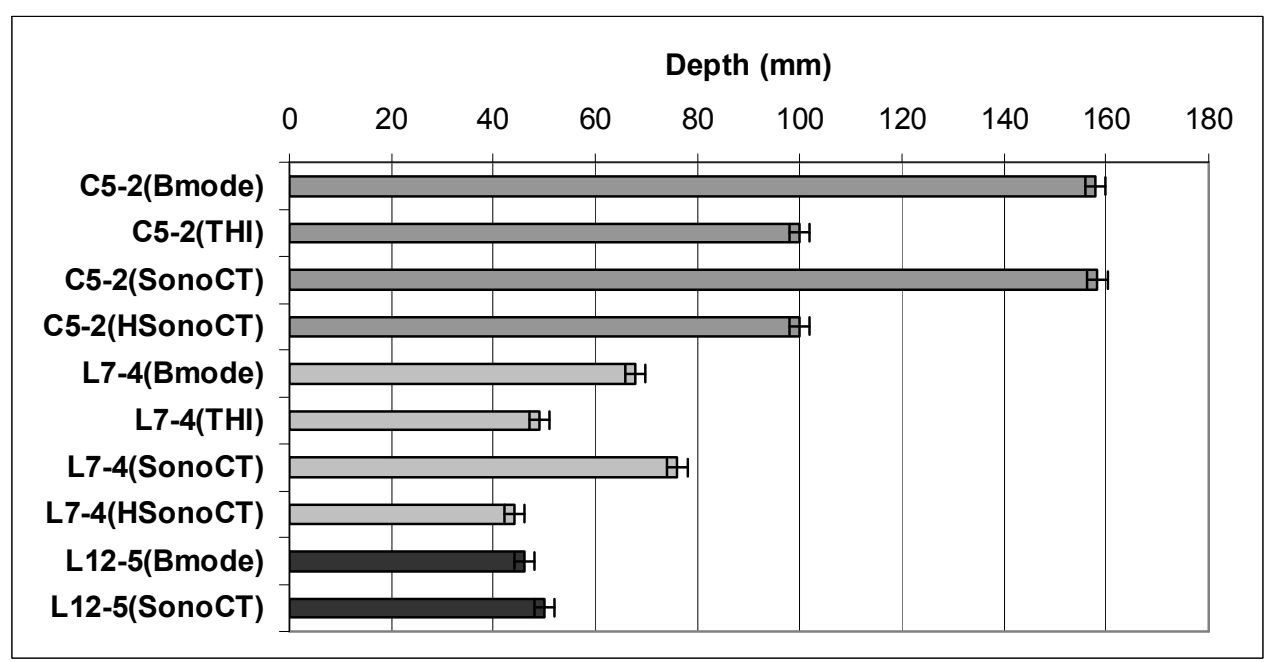

Fig. 13. 


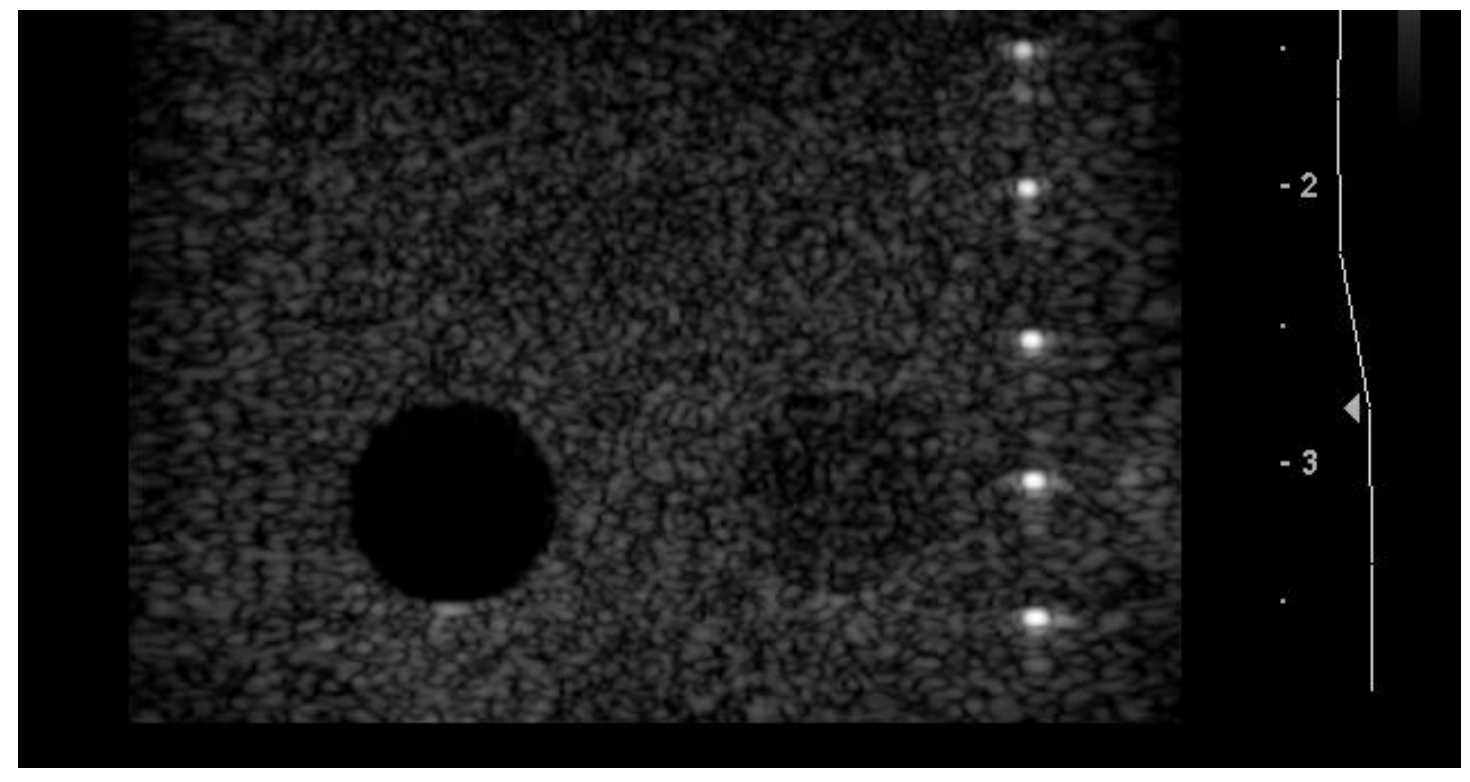

Fig. 14. 


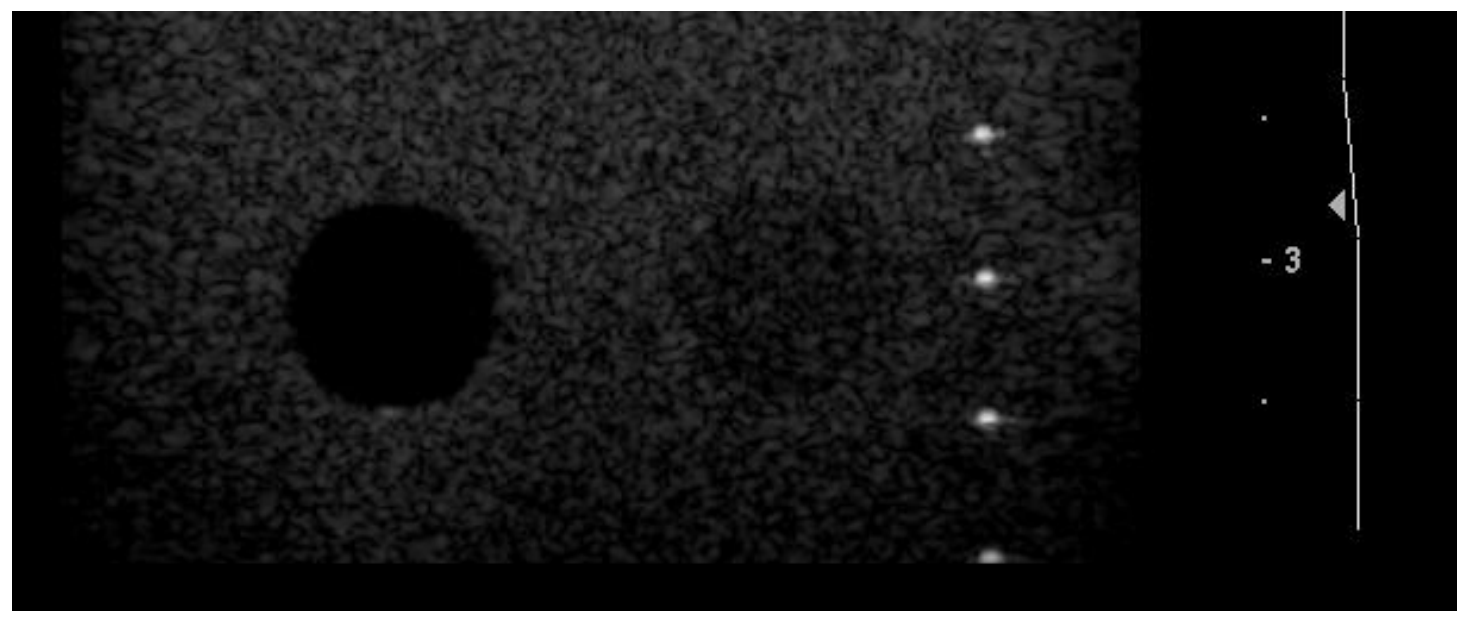

Fig. 15. 